\title{
Anomalous Diffusion in Gibbsian-Breaking of Wealth Distributions
}

\author{
Wan Abdullah W. A. T. and Khidzir S. M.* \\ Physics Department, Faculty of Sciences, University of Malaya, 50603 Kuala, Lumpur, Malaysia. \\ * sidiqmk@gmail.com. (Corresponding author). \\ Received on $19^{\text {th }}$ May 2010, accepted in revised form $22^{\text {nd }}$ July 2010.
}

\begin{abstract}
In a conservative system, energy equipartition results in a Boltzmann-Gibbs distribution. This is also expected in an ergodic kinetic economy if wealth is conserved. Empirical data however has shown the existence of a regime with a power law distribution. In models albeit with conservative wealth, we show that this arises due to anomalous diffusion.
\end{abstract}

(Keywords: Boltzmann-Gibbs, economy, wealth, anomalous diffusion)

PACS : 89.65.Gh, 02.30.Sa, 05.45.Pq

\section{INTRODUCTION}

Income distributions are observed to have a robust mixed distribution differentiating between the lower income and higher income groups. The distribution of income for the higher income group seems to fit a power law. Among the first observations of this is attributed to Pareto [1]. The income distribution for lower income group has been historically fitted to lognormal distributions, which is attributed to Champernowne [2] who also proposed a simple model describing the interactions.

Patriaca et al. [3] have proposed the Gamma distribution as a better fit for the lower income group drawing analogy to thermodynamics of a many body interacting system at equilibrium. It was put forth by Charterjee et al. in [4] that the Pareto exponent is observed to vary between 1 and 3 while the higher income group usually consists of less than $10 \%$ of the population. A recent review of the field was presented by Yakovenko et al. [12].

\section{STATISTICAL MECHANICS OF INCOME/WEALTH DISTRIBUTIONS}

It has been noted since Pareto that the distribution of income for the higher income group can be fitted to a power law. The distribution of income for the lower income group however has been fitted as either a lognormal distribution by Simon [5] and later by Montroll and Shlesinger [6] or as a Boltzmann-Gibbs distribution by Chatterjee [4] and Dragulescu [7]. Physicists attempting to model economic behavior adopt the analogy of large systems of interacting particles as seen in the kinetic theory of gases.
They hypothesized that the regular patterns observed in income and wealth distribution is due to a natural law for the statistical properties of many body systems interacting as an economy analogous to gases and liquids. Thus the description of an economy as a thermodynamic system allows the identification of the income distribution as the distribution of energy levels of particles in a gas.

The Boltzmann-Gibbs distribution states that the probability of finding a physical system in a state with energy is $\varepsilon$ given by

$P \mathbf{C}^{-}=c \mathrm{e}^{-c / 1}$

where $\mathrm{c}$ is the normalizing constant and $\mathrm{T}$ is the temperature.

In [7], the argument was that a many body interacting system such as the economy can be described by the Boltzmann-Gibbs distribution by choosing the conserved quantity as money. The process is described by the relation

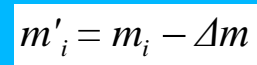

(2)

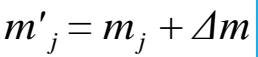

(3)

where agents $\mathrm{i}$ and $\mathrm{j}$ complete a transaction with the total amount of money before and after the transaction conserved.

The resultant wealth distribution from the interactions in (2) and (3) is able to account for the Boltzmann-Gibbs distribution. The trading model in [4] allows a distribution of wealth with a mixed distribution as observed in empirical data. The model is based on the assumptions made by Dragulescu [7], particularly conservation of money during a trade. The rules of trading can be written 
as

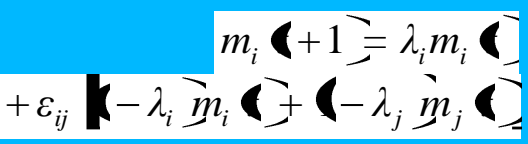

(4)

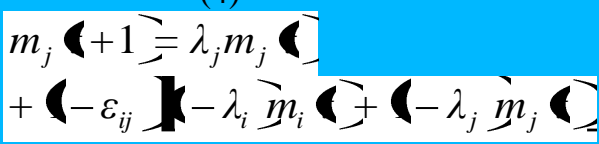

(5)

The model initializes $\lambda$ as an inhomogeneous parameter where $\lambda_{i}$ and $\lambda_{j}$ are saving propensities for agent $\mathrm{i}$ and $\mathrm{j}$ respectively.

These saving propensities are fixed over time, distributed independently, randomly and uniformly between $0 \leq \lambda \leq 1$

\section{ANOMALOUS DIFFUSION}

Anomalous diffusion is diffusion occurring at a faster or slower rate than normal. Experimentally, it is characterized by the scaling of the mean squared displacement with time given by

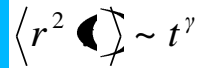

$\gamma$ is the scaling index for diffusion. $\gamma=1$ is normal diffusion, $\gamma>1$ is a superdiffusive process and $\gamma<1$ is a subdiffusive process.

An excellent example of the analysis is seen in the rotating annulus experiment [8].

Physical phenomena associated with anomalous diffusion is the occurence of long waiting times of a particle in a certain position and long jumps taken by a particle from one position to another.

To model systems that exhibit these phenomena, we employ the continuous time random walk (CTRW) model first introduced in [9].

It consists of successive random displacements $\Delta x_{n}$ and waiting times $\Delta t_{n}$ drawn from respective probability distribution functions

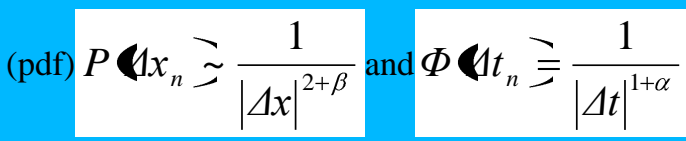

We are particularly interested in finding the position $x \in$ after time $t$ and its associated pdf $W \&, t$. For this, we adopt the inverse Fourier-Laplace Montroll-Weiss equation [10,11] given by

$W_{r} \ll, t \equiv t^{-\alpha / \beta} L_{\alpha, \beta} \mathbb{\&} / t^{\alpha / \beta}$

where $L_{\alpha, \beta}=Q \epsilon^{>}-\int d k E_{\alpha} \leftarrow k \uparrow-i k z$ is the universal scaling function. The spatio-temporal scaling can be obtained from this relation as

$$
X \underset{\sim}{\sim} t^{\alpha / \beta}
$$

$\alpha$ and $\beta$ represent the spatial and temporal exponents respectively in which $0<\beta<2$ and $0<\alpha<1$. The ratio $\alpha / \beta$ represents the interplay between super and subdiffusion where $\alpha / \beta \neq 0.5$ implies anomalous diffusion [11].

This relation was applied by Brockmann et. al. [11] to collected data from the website www.wheresgeorge.com where users could enter their location and bank note serial number into the website database and track the bank notes location over time as other users enter a bank notes serial number.
They show that movement of money is due to both scale-free periods of displacements and scale-free periods of rest as well.

\section{METHODOLOGY}

Anomalous diffusion has been observed in the 
diffusion of money across the United States. This observation has motivated us to study the diffusion of money in trading models. This was because of the observation by Brockmann as elaborated before, that the spatial inhomogeneities existing in a real economy in the form of geographical location and socioeconomic factors such as population density between geographic locations, will result in the observation of Levy flights in money displacement as well as long waiting times of money in a location.

A trading model is a suitable candidate for these observations to occur as the nature of a trading model, based on well defined trading rules will result in both a trapping of money with specific agents as well as more vibrant moving money with other agents. The Chakrabarti trading model is a suitable candidate to study anomalous diffusion in a trading model as the occurrence of long waiting times will naturally happen due to the existence of agents who have large saving propensities.

These agents will hold on to monies for longer periods of times due to its propensity to save more and trade less.

The Chakrabarti trading model has to be modified to allow proper calculations of jump lengths and waiting times. Only then can we attempt to perform diffusion analysis.

As the paradigm for our study is oriented towards the money in the trading model, we have to clearly define a path the money will flow to and also a proper mechanism to track the money's position and time at each location throughout the trading process. For the path the money will flow to, the clearly defined path will be determined by the agents trading money from one to another.

We have predetermined that agents can only trade with its immediate neighbors. In order to properly simulate this action, we firstly define a suitable lattice size of agents. Since our goal is to determine if the displacement is scale free, our lattice size must be at least 3 orders of magnitude in length. The lattice size is simply our definition of the area populated by the agents.

Once we have determined the lattice size we have to randomly choose an agent to perform a trade. This agent can only trade with its nearest neighbor. The definition of these neighbors is dependent on where the agent is located. There are three locations which determine who are the neighbors of an agent. These locations are the edges, the corners and the main lattices.

Since we want to study the diffusion of money, we have to know its location and time during each trade. In programming terms, this is accomplished by saving into an array a money's owners. Thus as the trading process goes on and money changes hands, the previous owners of a money will be noted and since we have defined the agents position as the spatial variable we have knowledge of the money's trajectory.

Similarly after a trade, the current iteration time will be saved into an array. Thus we currently have knowledge of time between one trade and another which allows us to calculate the waiting time between trades.

Now that we have stated the modifications we have made to track the money's position and time throughout the trading process, we can subsequently determine the displacements of each money in the system after a given period of time and also the waiting times between trades.

To calculate the displacements, we have to know after a certain period of time where the money currently is and what was it's original position.

We then make use of the determination of distance by applying the Pythagoras theorem. After we have obtained data that will allow us to plot the distribution of displacement, we want to obtain data that will allow us to plot the waiting time distribution. This is done by simply finding the time difference between successive jumps of money from one agent to another.

This data will then be sorted into bins in order to plot a distribution of waiting time. This is done by simply conducting a census of frequency of a waiting time over the entire waiting.

\section{RESULTS AND DISCUSSION}

In this section, we present the resultant PDF of displacement and its subsequent data analysis. We firstly present presenting a few PDFs of displacement after 4000 million iterations in the Figure 1.

As was reported by Brockmann, displacement between 1 to 10 units is also seen to scale linearly. This means there is an exponential growth in displacement during this range. Brockmann 
however did not report of a plateau as seen in displacements between 10 to 200 units.

We propose that these displacements are due to monies being in the vicinity of agents with a high saving propensity. Thus, these agents play the role of an attractor. This observation was not seen by Brockmann because displacements that were reported in wheresgeorge.com are of significant distance. Now that we have observed the change of the PDF of displacement with time, we want to perform a power law analysis to allow us to obtain the scaling exponent, $\beta$.

We present the portion of the distribution that we propose is scale free in Figure 2. We opt to sample 465 data points between 200 and 631 units of displacement as the scale free region.. To estimate $\beta$, we perform maximum limit estimation. We choose $x_{\min }$ as 200 .

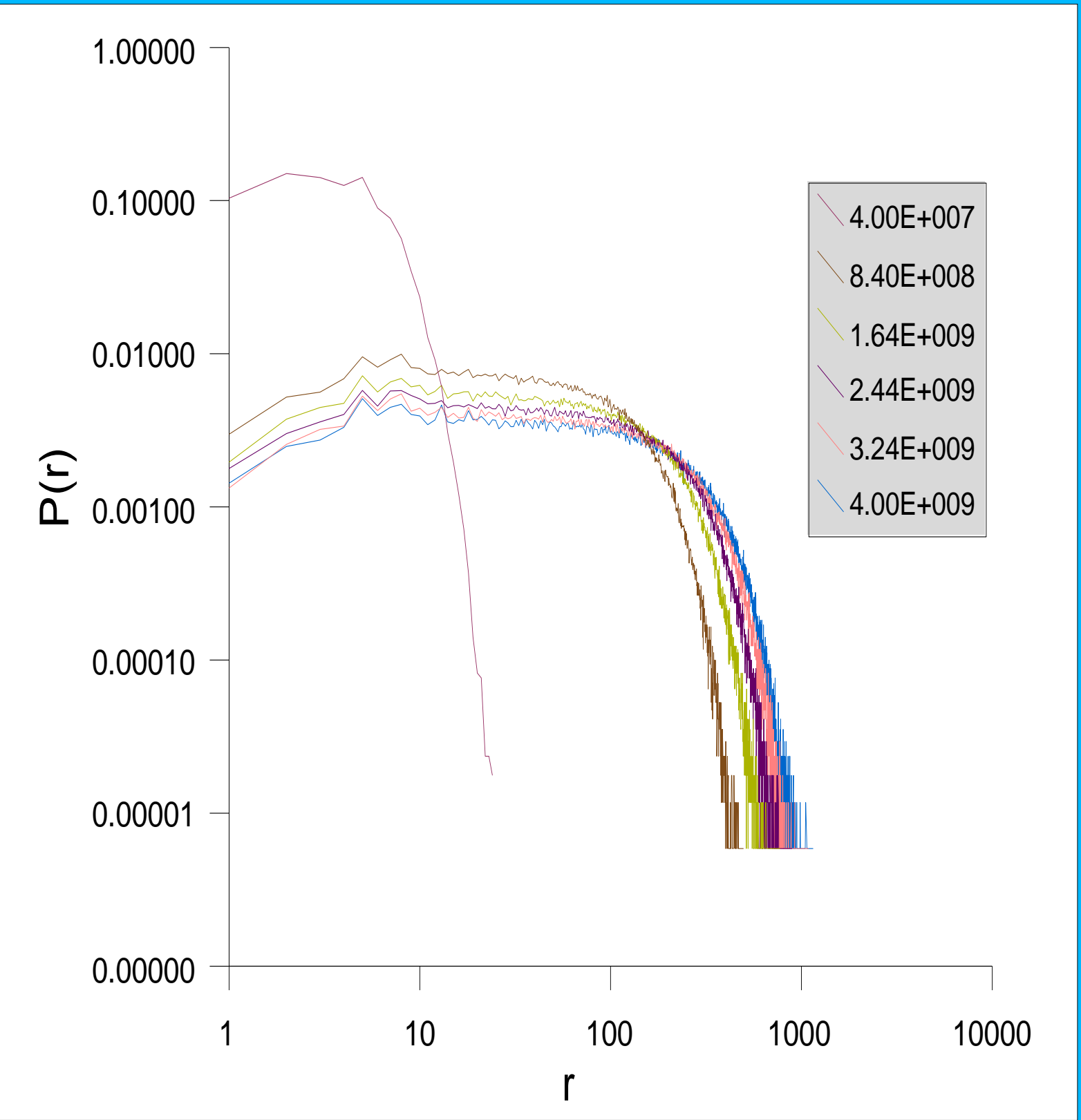

Figure 1: PDFs of displacement after 4000 million iterations 


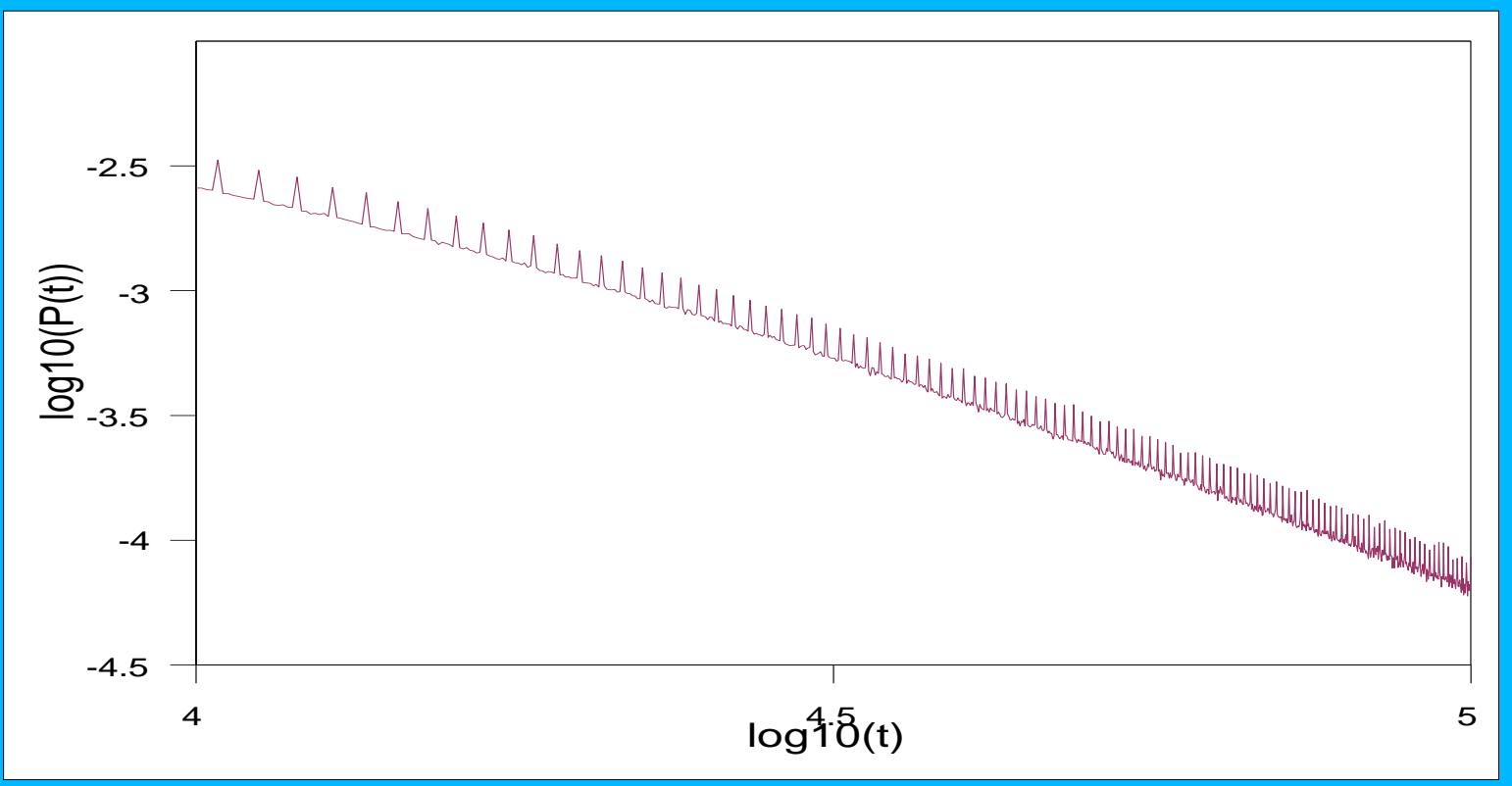

Figure 2: PDF of displacement between 200 and 631 units of displacement

We obtain the parameter estimate of the scaling exponent $\beta=2.4631$. This value suggests that money is undergoing superdiffusion. We then asses the goodness of fit of the parameter estimate of the scaling exponent with the Kolmogorov-Smirnov statistic also described in [12].

The value of $\mathrm{D}$ that we obtain is $\mathrm{D}=0.08$. The final step is to determine the p-value [12] for the obtained statistic. From the dataset of 464 points we randomly choose 400 data points to calculate the pvalue. We obtain a p-value of $92.0 \% \%$ which is an agreeable value.

Our next task will be to plot the distribution of waiting time of money with agents and infer the scaling exponent from the distribution. We show the PDF after $4{ }^{10}$ iterations in Figure 3.

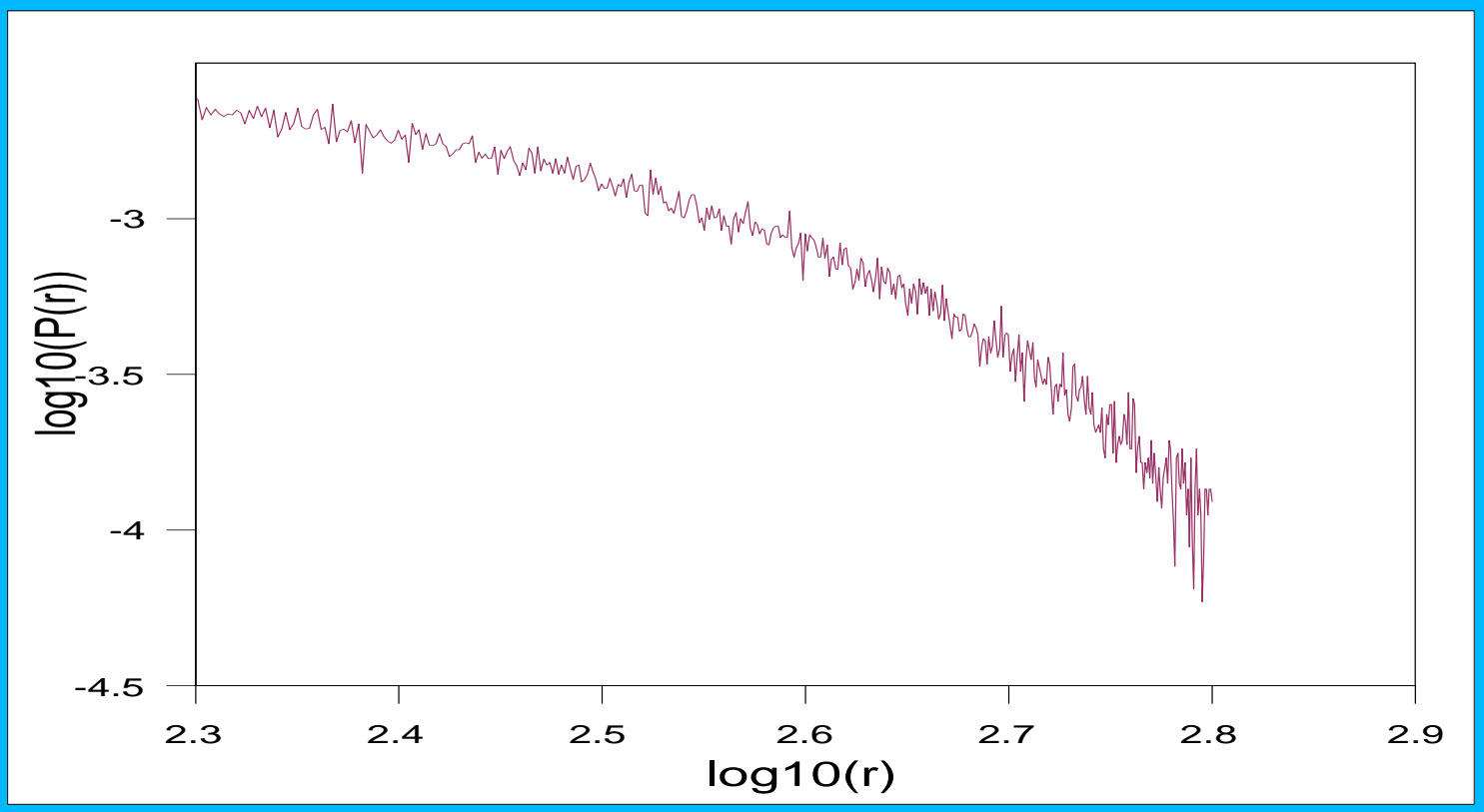

Figure 3: PDF of waiting time after 4000 million iterations 


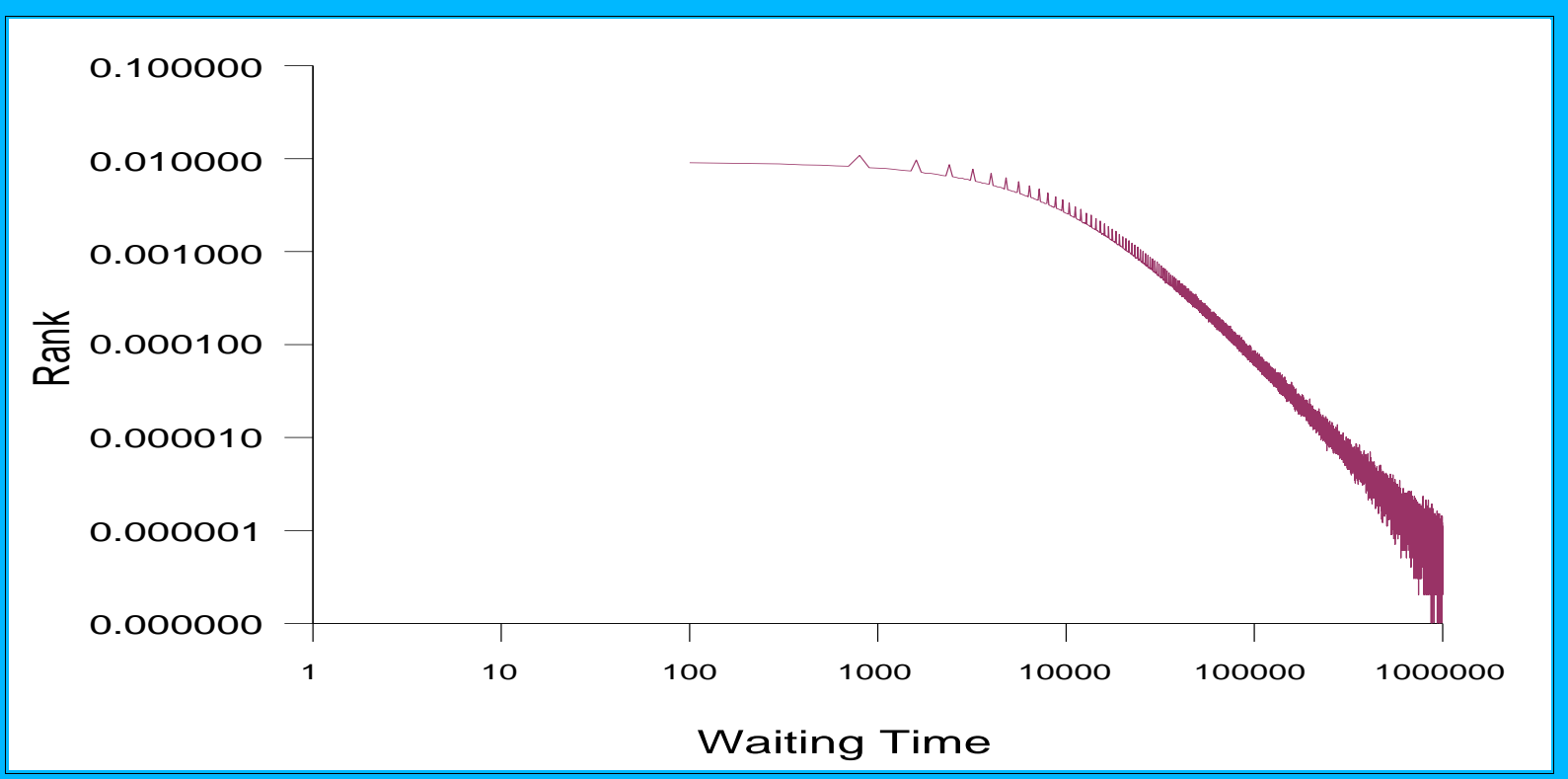

Figure 4: PDF of waiting time between 10000 and 100000 iterations

We firstly observe ripples in the distribution which is due to the biased random number generator used. The ripples can be reduced by applying a fairer random number generator. We then observe that half the portion of observations occurred under $10^{4}$ iterations.

There are however cases where monies have to wait longer than $10^{4}$ iterations and we hypothesize scale free behavior occurring in this region between $10^{4}$ and $10^{6}$ iterations. Our next step is to perform a power law analysis on the scale free region. We present the region in the Figure 4.

The first step is to perform a maximum likelihood estimation of the scaling exponent. We choose $x_{\min }$ as 4 in this case and the sample size is over 900 data points. We obtain the parameter estimate of the scaling exponent as $\alpha=1.6411$. This also suggests that the money is undergoing superdiffusion. We then asses the goodness of fit of the parameter estimate of the scaling exponent with the Kolmogorov-Smirnov statistic. The value of $D$ that we obtain is $\mathrm{D}=0.033$

Our final step is to determine the p-value for the obtained statistic. From the dataset of 900 points we randomly choose 700 data points to calculate the pvalue. We obtain a p-value of $91.0 \%$ which is an agreeable value.

\section{CONCLUSION}

A scale free observation for the pdf of money displacement and waiting time is seen for the Chakrabarti trading model. The ratio of $\alpha / \beta=1.50 \quad$ implies anomalous diffusion specifically superdiffusion.

\section{REFERENCES}

1. V.Pareto, Cours d'economie politique, Lausanne and Paris, 1897.

2. D. Champernowne, A model of income distribution, Economic Journal 63 (1953), 318351.

3. M. Patriaca, K. Kaski and A. Chakraborti, Statistical model with a standard Gamma distribution, Phys. Rev. E 70, 016104 (2004).

4. A. Chatterjee and B.K. Chakrabarti, Kinetic market models with single commodity having price fluctuations. Eur. Phys. J. B 54,399-404 (2006).

5. H.A Simon, On a class of skew distribution functions. Biometrika 42:3/4 (1955), 425-440

6. E.W Montroll and M.F. Shlesinger, Maximum Entropy Formalism, Fractals, Scaling 
Phenomena and 1/f Noise : A Tale of Tails. Journal of Statistical Physics 32 (1983), 209230.

7. A.A. Dragulescu and V.M. Yakovenko, Statistical mechanics of money, The European Physical Journal B 17 723-729.

8. T.H. Solomon, E.R. Weeks and H.L. Swinney, Observation of anomalous diffusion and Levy Flights in a two-dimensional rotating flow, Phys. Rev. Lett. 71, 3975 (1993).

9. E.W. Montroll and G.H. Weiss, Random walks on lattices, J. Math. Phys. 6,167-181(1965).
10. D. Brockmann, L. Hufnagel and T. Geisel, The scaling laws of human travel. Nature 439,462465 (2006).

11. D. Brockmann, Anomalous diffusion and the structure of human transportation networks. Eur. Phys. J. Special Topics 157,173-189 (2008).

12. V. Yakovenko and J. Barkley Rosser, Colloquium: Statistical mechanics of money, wealth, and income. Rev. Mod. Phys. 81 (2009). 